
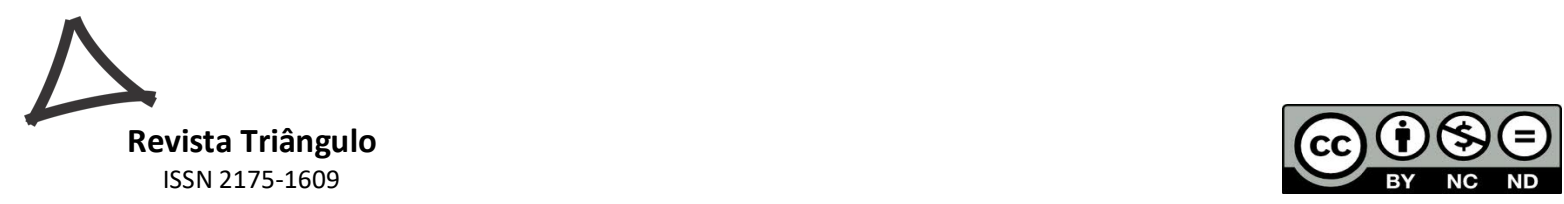

\title{
EMISSÕES DE GASES POLUENTES DA PRODUÇÃO SIMULTÂNEA DE BIODIESEL E HIDROGÊNIO
}

\author{
EMISSIONS OF POLLUTANT GASES FROM THE SIMULTANEOUS PRODUCTION OF \\ BIODIESEL AND HYDROGEN
}

\author{
EMISIONES DE GASES POLUENTES DE LA PRODUCCIÓN SIMULTÁNEA DE \\ BIODIESEL Y HIDROGENIO
}

\begin{abstract}
Ronney Arismel Mancebo Boloy. Centro Federal de Educação Tecnológica Celso Suckow da Fonseca (CEFET-RJ). E-mail: ronney.boloy@gmail.com

Gisele Maria Ribeiro Vieira Centro Federal de Educação Tecnológica Celso Suckow da Fonseca (CEFET-RJ). E-mail: gisele.cefetrj@gmail.com

Jaqueline Maria Ribeiro Vieira Centro Federal de Educação Tecnológica Celso Suckow da Fonseca (CEFET-RJ). E-mail: jaqueline.cefetrj@gmail.com

Jonni Guiller Ferreira Madeira Centro Federal de Educação Tecnológica Celso Suckow da Fonseca (CEFET-RJ). E-mail: gaiada123@hotmail.com

Marcus Val Springer Centro Federal de Educação Tecnológica Celso Suckow da Fonseca (CEFET-RJ). E-mail: marcusvalspringer@gmail.com

Marcelo Borges Rocha Centro Federal de Educação Tecnológica Celso Suckow da Fonseca (CEFET-RJ). E-mail: rochamarcelo36@yahoo.com.br
\end{abstract}

\begin{abstract}
RESUMO
O presente trabalho tem como objetivo quantificar o impacto ambiental da produção simultânea de biodiesel e hidrogênio. Estudos sobre a utilização de biodiesel e hidrogênio têm se mostrado promissores no ponto de vista da eficiência energética, mas é preciso atingir um maior potencial comercial. Aspectos econômicos e ambientais são considerados limitantes para o desenvolvimento tecnológico destes biocombustíveis. Entretanto, em se tratando de uma área em expansão, tonar-se necessário atrelar a essa tecnologia práticas efetivas de sensibilização ambiental tanto para as Instituições de Ensino Superior - IES, bem como para a população. Neste artigo, determinam-se as emissões de dióxido de carbono equivalente em cada etapa do processo produtivo: plantio, transporte, extração de óleo e produção de biodiesel e hidrogênio; seguindo o mecanismo de desenvolvimento limpo com biodiesel apresentado na convenção das nações unidas sobre mudança do clima (UNFCCC-2010). A etapa associada ao transporte foi a que apresentou menor emissão de dióxido de carbono equivalente, apenas $1 \%$ do valor total das emissões produzidas; já a etapa agrícola, produz $80 \%$ do valor total das emissões. O binômio biodiesel e hidrogênio representa uma inovação tecnológica, visto que do ponto de vista ambiental consegue-se uma redução das emissões do dióxido de carbono equivalente. Por isso, essa pesquisa contribui para a discussão acerca dos conceitos de inovação tecnológica e sustentabilidade levando em conta a qualidade ambiental dos processos produtivos e de seus produtos.
\end{abstract}

PALAVRAS-CHAVE: Emissões. Dióxido de carbono equivalente, Biodiesel, Hidrogênio. 


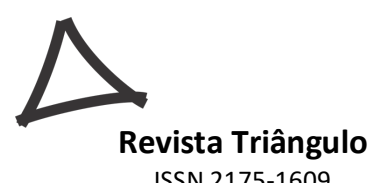

\section{ABSTRACT}

This study aims to quantify the environmental impact of simultaneous production of biodiesel and hydrogen. Studies on the use of biodiesel and hydrogen have shown promise in view of energy efficiency, but we must achieve a greater commercial potential. Economic and environmental aspects are considered as limiting for the technological development of these biofuels. However, in the case of an expanding area, it is necessary to link to this technology effective practices of environmental awareness for Higher Education Institutions and for the population. In this paper, we determined the equivalent carbon dioxide emissions at each stage of the production process: planting, transportation, oil extraction and production of biodiesel and hydrogen; following the clean development mechanism with biodiesel presented in the United Nations Convention on Climate Change (UNFCCC 2010). The step associated with the transport showed the lowest carbon dioxide equivalent emissions, only 1\% of the total amount of emissions produced; on the other hand, the agricultural stage produces $80 \%$ of the total emissions. The production of both biodiesel and hydrogen is a technological innovation, since that from the environmental point of view we achieved a reduction of the equivalent carbon dioxide emissions. Therefore, this research contributes to the discussion about the concepts of technological innovation and sustainability taking into account the environmental quality of the production processes and their products.

KEYWORDS: Emissions. Equivalent carbon dioxide, Biodiesel, Hydrogen.

\section{RESUMEN}

El presente trabajo tiene como objetivo cuantificar el impacto ambiental de la producción simultánea de biodiesel y hidrógeno. Los estudios sobre la utilización de biodiesel y hidrógeno son prometedores desde el punto de vista de la eficiencia energética, pero hay que alcanzar un mayor potencial comercial. Los aspectos económicos y ambientales se consideran limitantes para el desarrollo tecnológico de estos biocombustibles. Sin embargo, cuando se trata de un área en expansión, es necesario asociar a esa tecnología prácticas efectivas de sensibilización ambiental tanto para las Instituciones de Educación Superior - IES, así como para la población. En este artículo, se determinan las emisiones de dióxido de carbono equivalente en cada etapa del proceso productivo: plantación, transporte, extracción del aceite y producción del biodiesel y hidrógeno; de acuerdo com el mecanismo de desarrollo limpio con biodiesel presentado en la convención de las naciones unidas sobre cambio climático (UNFCCC-2010). La etapa asociada al transporte fue la que presentó menor emisión de dióxido de carbono equivalente, apenas el 1\% del valor total de las emisiones producidas; ya la etapa agrícola, produce el $80 \%$ del valor total de las emisiones. El binomio biodiesel y hidrógeno representa una innovación tecnológica, ya que desde el punto de vista ambiental se consigue una reducción de las emisiones del dióxido de carbono equivalente. Por eso, esta investigación contribuye para la discusión sobre los conceptos de innovación tecnológica y sostenibilidad teniendo en cuenta la calidad ambiental de los procesos productivos y de sus productos.

PALABRAS-CLAVE: Emisiones. Dióxido de carbono equivalente, Biodiesel, Hidrogeno.

\section{INTRODUÇÃO}

Nos últimos anos a produção de biocombustíveis tem se tornado um importante tema de pesquisa devido a suas aplicações em diversas áreas, principalmente nos setores de transporte e energia. Além disso, sua utilização traz benefícios ambientais, permitindo a redução das emissões de gases poluentes no meio ambiente. A utilização do biodiesel e do hidrogênio têm se mostrado relevante; recentemente um estudo de uma planta piloto para a produção de biodiesel a partir do óleo de girassol com alto conteúdo de ácido oléico, foi desenvolvido por Barontini et al. (2015) no estudo avalia-se o desempenho do óleo obtido a partir dos híbridos PR64 H31 e PR65 H41 de girassol e as propriedades do biodiesel obtido. 


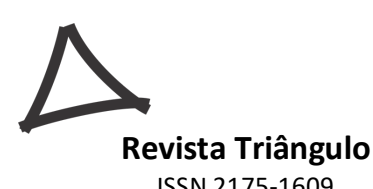

Em outro estudo, Song et al. (2015) realizaram uma revisão sobre a perspectiva global de recursos necessários para o desenvolvimento e utilização de algas marrons, como alternativa sustentável para a produção de biodiesel. Chen et al. (2015) fazem um estudo em forma de revisão que fornece as informações atualizadas sobre a produção de biocombustíveis à base de macroalgas, como biogás, bioetanol, biodiesel e bio-óleos, obtidos a partir de processos de digestão, fermentação, transesterificação, liquefação e técnica de pirólise respectivamente.

O hidrogênio é considerado o combustível do futuro e pode ser produzido a partir de processos biológicos (bio-hidrogênio) ou processos químicos (reforma). Chawlae Ghoshet at. (1992), apresentaram um estudo termodinâmico da produção de hidrogênio a partir da reforma do biogás, de modo a ser utilizado em uma célula combustível de ácido fosfórico. A produção de bio-hidrogênio a partir de lodo residual desintegrado e dissolvido por irradiação gama foi um estudo desenvolvido por Yin \& Wang (2015); o estudo mostra que esta irradiação em combinação com um pré-tratamento alcalino do lodo residual ativado, pode ser desintegrado e dissolvido para a produção de bio-hidrogênio. Oncelet al. (2015) apresentaram um estudo sobre a simulação das condições ao ar livre do efeito dos fatores ambientais nas cepas D1 e CC124 para a produção de hidrogênio.

Para tornar viável o uso de tecnologias na produção de biocombustíveis, e estes possam ser aproveitados em diversos setores, é preciso avaliar e diminuir seu impacto ambiental. Nesse sentido, a Educação Ambiental assume um papel relevante, visto que deve ser pensada como estratégia para compatibilizar o desenvolvimento econômico e a preservação do meio ambiente. De acordo com Jacobi (2003) a reflexão acerca de práticas sociais, dentro de um cenário marcado pela constante degradação ambiental, cria uma articulação necessária com a produção de sentidos sobre a Educação Ambiental. A produção simultânea de hidrogênio e biodiesel é um processo tecnológico proposto por Boloy (2014), no qual o referido autor avaliou a viabilidade técnica, econômica e ecológica da produção integrada de biodiesel e hidrogênio. Vários estudos têm sido desenvolvidos para avaliar o impacto ambiental da produção de biocombustíveis. Boloy et al. (2011) avaliaram e quantificaram o impacto ambiental do uso de gás de síntese em conjunto motor de combustão interna/gerador que funciona associado a um gaseificador de biomassa tipo downdraft . No estudo foram determinadas as emissões de gases poluentes e material particulado, além da eficiência ecológica do sistema proposto.

Coronado et al. (2010) avaliaram e quantificaram o impacto ambiental resultante da mistura do biodiesel (puro ou misturado ao diesel) e de combustão do diesel em usinas termelétricas que utilizam a tecnologia de ciclo combinado. Braga et al. (2013) apresentaram uma análise técnica, econômica e ecológica da produção de hidrogênio a partir da reforma do biogás. No estudo foi obtida uma eficiência ecológica do processo de 94,95\%. Dessa forma, a proposta tecnológica para produzir hidrogênio e biodiesel, de forma integrada, é categoricamente importante e fundamental na procura de processos que sejam energeticamente e ambientalmente sustentáveis. No presente trabalho é feito um estudo da quantificação e avaliação do impacto ambiental da produção simultânea de biodiesel e hidrogênio; assim como as implicações das emissões em cada etapa do processo. 


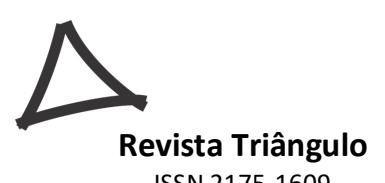

ISSN 2175-1609

\section{MATERIAIS E MÉTODOS}

No estudo utiliza-se óleo de canola como matéria prima para produzir biodiesel e hidrogênio, via reforma a vapor da glicerina. A Figura 1 mostra o ciclo de vida da canola, o qual começa com a produção de fertilizantes (nitrogenados, óxidos de fósforo $\left(\mathrm{P}_{2} \mathrm{O}_{5}\right)$ e óxidos de potássio $\left(\mathrm{K}_{2} \mathrm{O}\right)$, que são aplicados ao plantio da canola.

Figura 1 - Ciclo de vida da canola

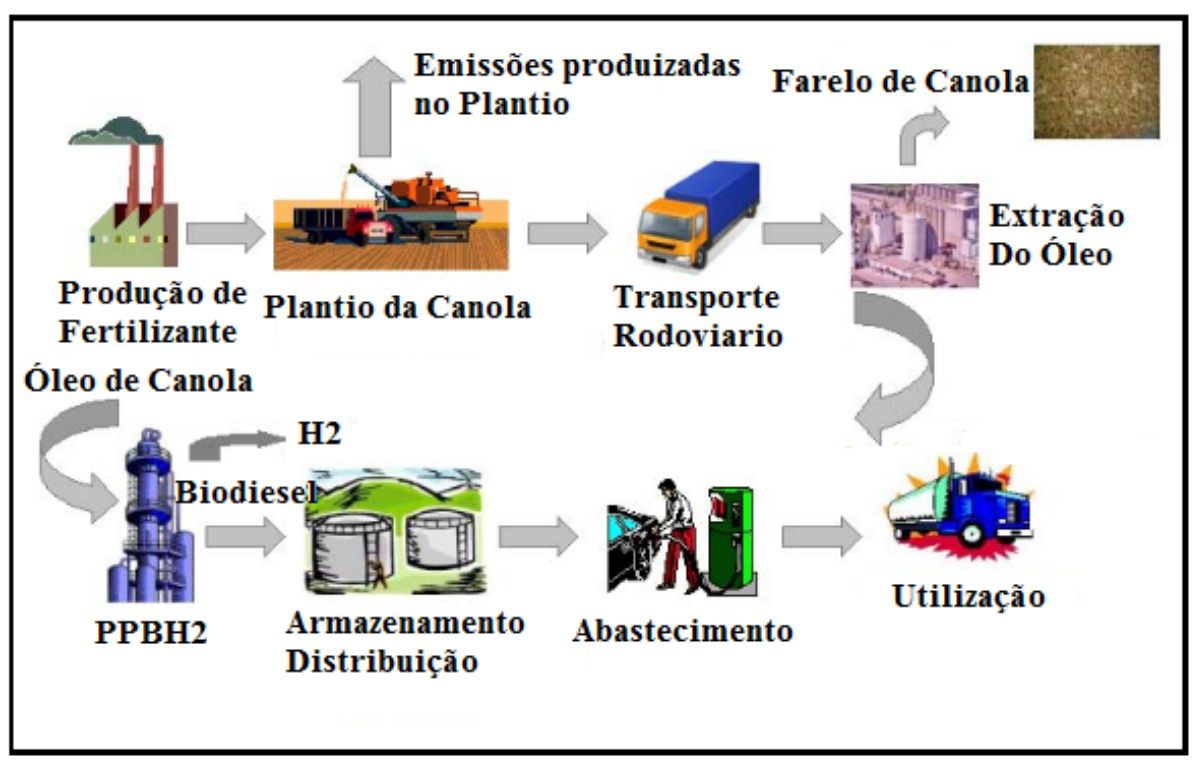

Fonte: Boloy, 2014.

Sua produção gera emissões que serão determinadas neste trabalho. Na etapa do plantio, são utilizadas máquinas agrícolas e caminhões para a coleta da canola e a preparação do solo. Essas máquinas produzem emissões que serão determinadas devido à combustão do diesel misturado com $5 \%$ de biodiesel. Na lavoura as emissões associadas à produção de herbicidas, inseticidas, assim como a utilização de fertilizantes também serão determinadas. No solo, existe uma quantidade de nitrogênio nos resíduos da lavoura e essa quantidade produz emissões diretas de óxidos de nitrogênio que serão calculadas. Além disso, a deposição de nitrogênio lixiviado e volatizado no solo produz emissões indiretas de óxidos de nitrogênio que também são determinadas. Os grãos de canola são transportados desde o plantio até a usina de extração de óleo através de transporte rodoviário (caminhões), sendo quantificadas as emissões associadas à combustão do diesel misturado com o biodiesel. Na usina, o óleo de canola bruto e o farelo de canola são obtidos como produto. Para a extração do óleo de canola, utiliza-se um solvente, geralmente o hexano, que possui alto rendimento. $\mathrm{O}$ processo de produção desse solvente, no entanto, gera emissões, as quais serão determinadas e incluídas no cálculo das emissões totais. Já o farelo de canola é produzido a partir dos grãos que passam por um processo de secagem e de aquecimento. Para isso se utiliza o vapor de água, que é produzido em caldeiras ou geradores a vapor que consomem diesel. A combustão 


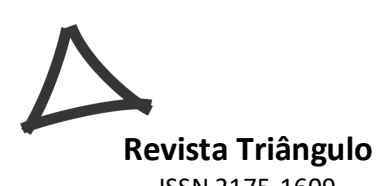

ISSN 2175-1609

do diesel em caldeiras produz emissões que também serão contabilizadas para a determinação das emissões totais. Há, ainda, outras emissões, como as produzidas pelo consumo de eletricidade fornecida pelo sistema interligado nacional (SIN), que também serão determinadas. O óleo de canola é utilizado na planta de biodiesel e hidrogênio para produzir: biodiesel, glicerina e hidrogênio. A planta gera emissões pela utilização do óleo, catalisadores, metanol, vapor de água e da eletricidade, essas emissões também serão determinadas neste item. Finalmente, o biodiesel é armazenado e distribuído para sua utilização no setor do transporte ou, também, no setor energético.

As emissões das etapas de produção e transporte das sementes até o plantio não são consideradas no estudo, devido à dificuldade para estabelecer os fatores de emissão dessas etapas. As emissões associadas ao transporte dos insumos utilizados na lavoura não são estimadas no estudo devido à dificuldade de obtenção dessas informações.

De acordo com a metodologia da Convenção das Nações Unidas sobre Mudança do Clima, as emissões de dióxido de carbono de origem biológica não são consideradas, uma vez que essa metodologia somente estipula as emissões produzidas pela mudança de estoque de carbono do solo quando há prática de manejo. No presente trabalho, essas emissões também não serão levadas em consideração.

$\mathrm{Na}$ fase industrial, as emissões associadas ao consumo de eletricidade do SIN são consideradas nas etapas de extração de óleo e na planta de produção de biodiesel e hidrogênio. Na Tabela 1, mostra-se o fator de emissão de eletricidade por mês durante o ano 2014. Nesse estudo para os cálculos das emissões foi utilizado o valor médio.

Tabela 1- Fator de emissão da produção de eletricidade no Brasil

Fator de Emissão da Produção de Eletricidade

\begin{tabular}{cc}
\hline & Fator Médio Anual $\left(\mathrm{tCO}_{2 \mathrm{e}} / \mathrm{MWh}\right)$ \\
Mês & Valor \\
Janeiro & 0,0911 \\
Fevereiro & 0,1169 \\
Março & 0,1238 \\
Abril & 0,131 \\
Média & 0,1157 \\
\hline
\end{tabular}

Fonte: Boloy, 2014.

As emissões associadas ao transporte dos insumos nas etapas de extração de óleo e produção de biodiesel não são consideradas. $O$ estudo considera a usina de extração do óleo junto à usina de produção de biodiesel e hidrogênio. O transporte agrícola até a usina de extração do óleo de canola é realizado através de caminhões. Sendo assim, considera-se o consumo de combustível, distância percorrida e a capacidade de carga do caminhão. Finalmente, a 


\section{Revista Triângulo}

ISSN 2175-1609

metodologia adotada não considera, em nenhuma das etapas, as emissões associadas à fabricação de máquinas e equipamentos e à construção da usina de extração de óleo e planta de produção de biodiesel e hidrogênio. Para a determinação das emissões de dióxido de carbono equivalente é utilizada a seguinte equação na forma geral:

$$
C O_{2 i} e=\sum_{i=1}^{n}\left(Q_{i} * F E_{i}\right) C O_{2 i} e=\sum_{i=1}^{n}\left(Q_{i} * F E_{i}\right)
$$

Em que:

$\mathrm{CO}_{2 \mathrm{ie}}$ - Dióxido de carbono equivalente do produto i utilizados na etapa i (kg CO2kg óleo)

$\mathrm{Q}_{\mathrm{i}}$ - Quantidade do produto i utilizado na etapa $\mathrm{i}$. Ei- Fator de emissão do produto i $\mathrm{t}$ $\mathrm{CO}_{2 \mathrm{e}} / \mathrm{t}_{\mathrm{i}}$.

A equação 2 permite a determinação das emissões produzidas pela combustão do diesel nas máquinas agrícolas. É importante destacar que o cálculo considera a venda do diesel com $5 \%$ de biodiesel. Além disso, considera-se o biodiesel produzido pela rota metílica, a mais utilizada no Brasil. O fator RMetanol considera a percentagem de metanol no biodiesel, cujo valor é desprezível na composição do biodiesel produzido nesta planta.

$$
\begin{aligned}
& \text { CO2 } e_{\text {Comb-Dissel-Máq.Agri }}=\left(Q_{\text {Dissel-Agri }} * 0,95 * F E_{\text {Comb-Diesel }}\right)+\left(Q_{\text {Dissel-Agri }} * 0,05 *\right. \\
& \left.F E_{\text {Comb-Metanol }} * R_{\text {Metanol }}\right) \\
& \text { CO2 } e_{\text {Comb-Dissel-Máq.Agri }}=\left(Q_{\text {Diessl-Agri }} * 0,95 * F E_{\text {Comb-Diesel }}\right)+\left(Q_{\text {Diesel-Agri }} * 0,05 *\right. \\
& \left.F E_{\text {Comb-Metanol }} * R_{\text {Metanol }}\right)
\end{aligned}
$$

A equação 3, permite a determinação da quantidade de emissões de gases do efeito estufa produzida pela aplicação de fertilizantes nitrogenados sintéticos e orgânicos na lavoura da canola. No Brasil, a utilização de fertilizante orgânico nos cultivos de oleaginosas é praticamente nulo. Como não existe uma especificação do tipo de fertilizante nitrogenado aplicado, e como a uréia apresenta 45\% de nitrogênio na sua composição (CNPT, 2014), considerou-se a aplicação da metade de nitrogênio vindo da uréia, o que resultou uma quantidade de $0,048 \mathrm{t} /$ hectare de uréia aplicada ao solo.

$$
\begin{aligned}
& \text { CO } 2 e_{\text {Frrt }-N}=\left(Q_{N-\text { Sint }} * \frac{44 * 298}{28} * F E_{N 2 O-N}\right)+\left(Q_{\text {Urbia }} * F E_{\text {CO2Apli-Uroia }}\right) \\
& \text { CO2 } e_{\text {Frrt }-N}=\left(Q_{N-\operatorname{Sint}} * \frac{44 * 298}{28} * F E_{N 2 O-N}\right)+\left(Q_{\text {Ureia }} * F E_{\text {CO2Apli-Ureia }}\right)
\end{aligned}
$$

A equação 4 permite a determinação das emissões de gases do efeito estufa produzidas pela manejo da lavoura em forma de $\mathrm{N}_{2} \mathrm{O}$

. Já a equação 5 é utilizada para calcular a quantidade de nitrogênio nos resíduos da lavoura que retornam ao solo.

$$
\begin{gathered}
C O 2 e_{N 2 O-D i r}=\left(\left(Q_{C R}+Q_{M O S}\right) * F E_{N 2 O-N}\right) * \frac{44 * 298}{28} \\
C O 2 e_{N 2 O-D i r}=\left(\left(Q_{C R}+Q_{M O S}\right) * F E_{N 2 O-N}\right) * \frac{44 * 298}{28}
\end{gathered}
$$


Revista Triângulo

ISSN 2175-1609

$$
\begin{array}{r}
Q_{C R}=C R O P * F R A C_{\text {Renovada }} *\left[R_{A G} * N_{A G} *\left(1-F R A C_{\text {Removida }}\right)+R_{B G} * N_{B G}\right] \\
Q_{C R}=C R O P * F R A C_{\text {Renovada }} *\left[R_{A G} * N_{A G} *\left(1-F R A C_{\text {Removida }}\right)+R_{B G} * N_{B G}\right]
\end{array}
$$

\section{RESULTADOS E DISCUSSÃO}

No Brasil o Programa Nacional de Produção de Biodiesel - PNPB tem como objetivo reduzir a emissão de GEE (gases do efeito estufa) substituindo um percentual do diesel pelo biodiesel, significando a substituição de um combustível fóssil por um combustível renovável. Além disso, outro aspecto relevante do PNPB é a inclusão social e o desenvolvimento regional gerando emprego e renda através do plantio de oleaginosas pela agricultura familiar. Nesse contexto a determinação das emissões do GEE, cuja medida é quantificada em toneladas de dióxido de carbono equivalente $\left(\mathrm{tCO}_{2 \mathrm{e}}\right)$ é importante no intuito de mitigar o impacto ambiental destas emissões na atmosfera. Cada tonelada de dióxido de carbono equivalente que seja reduzida na atmosfera é equivalente a uma unidade emitida pelo Conselho Executivo do Mercado de Desenvolvimento Limpo - MDL, também chamado como Redução Certificada de Emissão (RCE). Nesse sentido o objetivo do MDL é oferecer um 1 crédito de carbono para cada tonelada de $\mathrm{CO}_{2}$ e retirada ou deixada de emitir para a atmosfera.

A Tabela 2 mostra as emissões produzidas por cada insumo em relação a seu fator de emissão na etapa da lavoura da canola. No cultivo da canola não é utilizada a cal, o que justifica a adoção de valor zero para a quantidade de cal aplicada ao solo. Aplicando a Equação 1, o valor das emissões de gases do efeito estufa na etapa de lavoura para a canola é 1,41 $\mathrm{tCO}_{2 \mathrm{e}} /$ hectare.

Tabela 2 - Quantidades de emissões produzidas pelos principais insumos utilizados na etapa da lavoura do óleo

\begin{tabular}{|c|c|c|}
\hline Parâmetro(*) & UNIDADE & Valor \\
\hline $\mathrm{Q}_{\mathrm{N}-\mathrm{Sint}}$ & t N.hectare-1 & 0,06 \\
\hline $\mathrm{FE}_{\text {ProdN }}$ & $\mathrm{t} \mathrm{CO} 2 \mathrm{e} . \mathrm{tN}-1$ & 3,97 \\
\hline QP2O5 & $\mathrm{t}_{2} \mathrm{O}_{5}$.hectare-1 & 0,06 \\
\hline $\mathrm{FE}_{\text {prodP2O5 }}$ & $\mathrm{t} \mathrm{CO}_{2 \mathrm{e}} . \mathrm{tN}-1$ & 0,33 \\
\hline $\mathrm{Q}_{\mathrm{K} 2 \mathrm{O}}$ & t $\mathrm{K}_{2} \mathrm{O}$.hectare-1 & 0,03 \\
\hline FE $_{\text {ProdK2O }}$ & t CO $2 \mathrm{e} . \mathrm{tK} 2 \mathrm{O}-1$ & 0,4 \\
\hline $\mathrm{Q}_{\mathrm{cal}}$ & t cal.hectare-1 & 0 \\
\hline FEProdCal & t CO 2 e.tCal-1 & 0 \\
\hline $\mathrm{Q}_{\text {diesel }}$ & t Diesel.hectare-1 & 0,05453 \\
\hline FE ProdDiesel & $\mathrm{t} \mathrm{CO}_{2 \mathrm{e} . \mathrm{tDiesel}-1}$ & 0,32 \\
\hline Qherb & Therb.hectare-1 & 0,01 \\
\hline FE $E_{\text {Prodherb }}$ & t CO2e.therb-1 & 25 \\
\hline$Q_{\text {inse }}$ & Tinse.hectare-1 & 0,03 \\
\hline FE Prodins & t CO2e.tins-1 & 29 \\
\hline CO2eProd-Ins-Agri & t coze.hectare-1 & 1,41 \\
\hline
\end{tabular}
de canola 


\section{Revista Triângulo}

ISSN 2175-1609

Fonte: Boloy, 2014.

(*) - A representação de cada um dos parâmetros encontra-se no apêndice deste artigo.

A Tabela 3 mostra as emissões produzidas pela combustão do diesel nas máquinas agrícolas. Considerando os fatores de emissão para a combustão do diesel e do etanol, assim como a vazão do diesel o valor das emissões de gases do efeito estufa obtido é $0,18 \mathrm{tCO}_{2 \mathrm{e}} /$ hectare.

Tabela 3- Emissões produzidas pela combustão do diesel nas máquinas agrícolas

\begin{tabular}{ccc}
\hline Parâmetro(*) & UNIDADE & Valor \\
\hline $\mathrm{FE}_{\text {combdiesel }}$ & $\mathrm{tCO}_{2 \mathrm{e}} \cdot \mathrm{tcombdiese}-1$ & 3,498 \\
$\mathrm{FE}_{\text {combmetanol }}$ & $\mathrm{tCO}_{2 \mathrm{e} \cdot \text { tmetanol-1 }}$ & 1,375 \\
$\mathrm{Q}_{\text {diesel-agri }}$ & tdiesel.hectare-1 & 0,05453 \\
$\mathbf{C O 2}_{\text {comb-diesel-agri }}$ & $\mathbf{t C O}_{\mathbf{2}}$.hectare-1 & $\mathbf{0 , 1 8}$
\end{tabular}

Fonte: Boloy, 2014.

(*) - A representação de cada um dos parâmetros encontra-se no apêndice deste artigo.

A Tabela 4 mostra as emissões de gases do efeito estufa produzida pela aplicação de fertilizantes nitrogenados sintéticos e orgânicos na lavoura da canola. Desta forma, o valor das emissões de gases do efeito estufa é $0,32 \mathrm{tCO}_{2 \mathrm{e}} /$ hectare.

Tabela 4 - Emissões de gases do efeito estufa produzidas pela aplicação de fertilizantes nitrogenados sintéticos e

\begin{tabular}{|c|c|c|}
\hline Parâmetro(*) & UNIDADE & Valor \\
\hline$Q_{\text {ueria }}$ & t ureia.hectare-1 & 0,048 \\
\hline $\mathrm{FE}_{\mathrm{CO} \text { 2apli-uréia }}$ & $\mathrm{t} \mathrm{CO}_{2} / \mathrm{t}$ ureia- 1 & 0,733 \\
\hline $\mathrm{FE}_{\mathrm{N} 2 \mathrm{O}-\mathrm{N}}$ & $\mathrm{t} \mathrm{N}_{2} \mathrm{O}-\mathrm{N} / \mathrm{t} \mathrm{N}-1$ & 0,01 \\
\hline $\mathrm{CO} 2 \mathrm{e}_{\text {fert-N }}$ & $\mathrm{t} \mathrm{CO}_{2 \mathrm{e}} /$ hectare-1 & 0,32 \\
\hline
\end{tabular}

Fonte: Boloy, 2014.

(*) - A representação de cada um dos parâmetros encontra-se no apêndice deste artigo.

A Tabela 5 mostra as emissões de gases do efeito estufa produzidas pela manejo da lavoura em forma de $\mathrm{N}_{2} \mathrm{O}$ (equação 4). Para o cálculo das emissões é importante conhecer a quantidade de nitrogênio nos resíduos da lavoura que retornam ao solo (equação 5). O valor das emissões de gases do efeito estufa, produto das emissões diretas de $\mathrm{N} 2 \mathrm{O}$ determinado, é 0,40 t $\mathrm{CO}_{2 \mathrm{e}} /$ hectare.

Tabela 5- Emissões diretas de $\mathrm{N}_{2} \mathrm{O}$ produzidas pelo manejo da lavoura

\begin{tabular}{ccc}
\hline Parâmetro $(*)$ & UNIDADE & Valor \\
\hline $\mathrm{D}$ & \% biomassa seca na canola & 0,9 \\
$\mathrm{R}_{\mathrm{ag}}$ & - & 2 \\
$\mathrm{~N}_{\mathrm{ag}}$ & t N.t matéria seca-1 & 0,005 \\
\hline
\end{tabular}




\begin{tabular}{|c|c|c|}
\hline \multicolumn{2}{|c|}{$\begin{array}{l}\text { Revista Triângulo } \\
\text { ISSN 2175-1609 }\end{array}$} & (c) $\underset{\mathrm{BY}}{(\mathrm{NC}} \Theta_{\mathrm{ND}}$ \\
\hline Frac $_{\text {renovada }}$ & - & 0 \\
\hline $\mathrm{Rbg}$ & - & 1,73 \\
\hline CROP & t biomassa seca.hectare-1 & 1,98 \\
\hline Nbg & t N.t matéria seca-1 & 0,022 \\
\hline Rbg-bio & - & 0,4 \\
\hline Qcr & t N.hectare-1 & 0,02554 \\
\hline Qmos & t N.hectare-1 & 0,06 \\
\hline $\mathrm{FE}_{\mathrm{N} 2 \mathrm{O}-\mathrm{N}}$ & t $\mathrm{N}_{2} \mathrm{O}-\mathrm{N} . \mathrm{t} \mathrm{N}-1$ & 0,01 \\
\hline $\mathrm{CO}_{2 \mathrm{eN2Odir}}$ & t CO 2 e.hectare-1 & $\mathbf{0 , 4 0}$ \\
\hline
\end{tabular}

Fonte: Boloy, 2014.

(*) - A representação de cada um dos parâmetros encontra-se no apêndice deste artigo.

A Tabela 6 apresenta as emissões indiretas de $\mathrm{N}_{2} \mathrm{O}$ no manejo da lavoura da canola. Essas emissões são a soma da deposição de nitrogênio lixiviado e volatizado. Segundo Carvalho (2012), para a determinação das emissões do nitrogênio lixiviado é necessário conhecer a quantidade de nitrogênio sintético e o nitrogênio que retorna ao solo. Para o cálculo do volatizado, é importante conhecer a quantidade de nitrogênio sintético. Sendo assim, o valor das emissões indiretas de $\mathrm{N}_{2} \mathrm{O}$, no manejo da lavoura da canola, é 0,12 t $\mathrm{CO}_{2 \mathrm{e}}$ /hectare. Finalmente, pode ser conhecido o valor total das emissões de gases do efeito estufa da etapa da agricultura ou plantio: 2,42 t CO2e/hectare ou 3,002 t CO2e/t biodiesel. A Tabela 7 mostra as emissões associadas ao transporte entre a etapa agrícola e a etapa industrial (extração de óleo e produção de biodiesel e hidrogênio). $O$ cálculo considera o consumo médio de combustível do caminhão assim como a distância percorrida e, também, a carga nas viagens de ida e volta. Além disso, também será considerado o rendimento de extração do óleo refino e do óleo bruto, assim como fatores adimensionais, relacionando a quantidade do biodiesel produzido ao óleo de canola. $\mathrm{O}$ valor das emissões produzidas na etapa do transporte é 0,050 t CO2 2 /biodiesel.

Tabela 6 - Emissões indiretas de $\mathrm{N}_{2} \mathrm{O}$ no manejo da lavoura de canola

\begin{tabular}{|c|c|c|c|}
\hline Parâmetro(*) & UNIDADE & Valor & Fonte \\
\hline $\mathrm{FRAC}_{\text {sint }}$ & $\begin{array}{l}\text { t N Volatizado.t } \mathrm{N} \\
\text { aplicado-1 }\end{array}$ & 0,1 & - \\
\hline $\mathrm{Q}_{\mathrm{N}-\mathrm{Sint}}$ & t N aplicado.há-1 & 0,06 & (Gazzoni et al., 2009) \\
\hline $\mathrm{FE}_{\mathrm{vol}}$ & t N 2 O-N.t N-1 & 0,01 & (Buratti et al., 2010) \\
\hline $\mathrm{CO} \mathrm{e}_{\mathrm{N} 2 \mathrm{O}-\mathrm{V} \text { olat }}$ & t $\mathrm{N}_{2} \mathrm{O}$-N.ha-1 & 0,00006 & - \\
\hline FRAC lixivação & t N lixiviado.t N-1 & 0,3 & - \\
\hline $\mathrm{FE}_{\text {lixivação }}$ & $\begin{array}{c}\mathrm{t} \mathrm{N}_{2} \mathrm{O}-\mathrm{N} \text {.t lixiviado- } \\
1\end{array}$ & 0,0075 & (Buratti et al., 2010) \\
\hline CO2 $\mathrm{e}_{\mathrm{N} 2 \mathrm{Olixiviado}}$ & t $\mathbf{N}_{2} \mathrm{O}-\mathrm{N}$. ha-1 & 0,000192 & - \\
\hline $\mathrm{CO}_{\mathrm{N} 2 \mathrm{O}} \mathrm{e}_{\mathrm{ndir}}$ & t CO $\mathrm{CO}_{2 \mathrm{e}} \mathrm{ha-1}$ & 0,12 & - \\
\hline
\end{tabular}

Fonte: Própria.

(*) - A representação de cada um dos parâmetros encontra-se no apêndice deste artigo. 


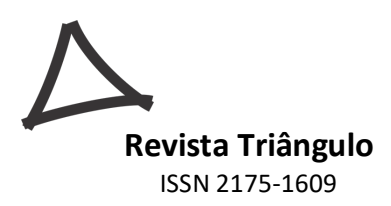

Tabela 7- Emissões associadas ao transporte entre a etapa de agricultura e industrial

\begin{tabular}{|c|c|c|c|}
\hline Parâmetro(*) & UNIDADE & Valor & Fonte \\
\hline $\mathrm{FE}_{\text {combdieselrodov }}$ & $\mathrm{t} \mathrm{CO}_{2 \mathrm{e}} . \mathrm{t}$ diesel-1 & 3,20 & (Carvalho, 2012) \\
\hline $\begin{array}{l}\text { Distância } \\
\text { percorrida } \\
\text { (ida e volta) }\end{array}$ & $\mathrm{Km}$ & 40 & (Gazzoni et al., 2009) \\
\hline $\begin{array}{l}\text { Cons. Comb. } \\
\text { Media }\end{array}$ & km.1-1 & 2,25 & (Gazzoni et al., 2009) \\
\hline Carga & tgrãos.canola-1 & 2,46 & - \\
\hline Produtividade & g biod.kg óleo-1 & 963,67 & - \\
\hline $\begin{array}{l}\text { Massa específica } \\
\text { do Diesel }\end{array}$ & kg.m-3 & 852 & - \\
\hline 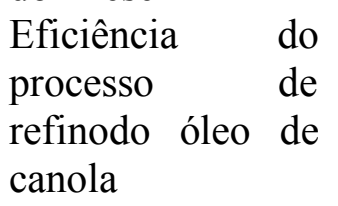 & $\begin{array}{l}\text { k refino.kg óleo } \\
\text { cru-1 }\end{array}$ & 0,97 & (Buratti et al., 2010) \\
\hline $\mathrm{FE}_{\text {prod-diesel }}$ & t $\mathrm{CO}_{2 \mathrm{e} .} \mathrm{t}$ diesel-1 & 0,32 & (Carvalho, 2012) \\
\hline FE combdieseltransp & t CO $2 \mathrm{e} . \mathrm{t}$ diesel & 3,14 & (Carvalho, 2012) \\
\hline $\mathrm{FE}_{\text {combmetanol }}$ & $\mathrm{t} \mathrm{CO}_{2 \mathrm{e} .} . \mathrm{t}$ metanol-1 & 1,38 & (Carvalho, 2012) \\
\hline CO2 e transp-agri-ind & t $\mathrm{CO}_{2 \mathrm{e} .} . \mathrm{t}$ biodiesel-1 & $\mathbf{0 , 0 5 0}$ & - \\
\hline
\end{tabular}

Fonte: Própria.

(*) - A representação de cada um dos parâmetros encontra-se no apêndice deste artigo.

A Tabela 8 mostra as emissões produzidas na etapa de extração do óleo de canola. Nesta etapa, são considerados o consumo de eletricidade proveniente do Sistema Nacional Interligado (SNI), assim como os fatores de emissão associados ao consumo. Considerou-se também a utilização de hexano como solvente para o processo de extração, pois, segundo Carvalho (2012), este solvente tem sido estudado por muitos autores e apresenta o melhor rendimento na extração.Finalmente, o processo de extração de óleo necessita do vapor, que é gerado em caldeiras que queimam diesel. Portanto, as emissões inerentes ao processo de combustão também são calculadas. A quantidade de emissões produzidas no processo de extração do óleo de canola é 0,06 t $\mathrm{CO}_{2 \mathrm{e}} / \mathrm{t}$ biodiesel e as emissões do processo de combustão do diesel em caldeira é $0,28 \mathrm{t} \mathrm{CO}_{2 \mathrm{e}} / \mathrm{t}$ biodiesel.

A Tabela 9 mostra as emissões associadas ao processo de produção de biodiesel e hidrogênio.No cálculo, serão considerados o consumo de eletricidade fornecido pelo Sistema Nacional Interligado e os fatores de emissão inerentes a este consumo, assim como as emissões de dióxido de carbono equivalente produzidas na combustão do gás natural para a geração de vapor. $\mathrm{O}$ valor das emissões do processo de produção de biodiesel e glicerina é $0,35 \mathrm{t} \mathrm{CO}_{2 \mathrm{e}} / \mathrm{t}$ biodiesel. 


Revista Triângulo
ISSN 2175-1609

Fonte: Própria.

(*) - A representação de cada um dos parâmetros encontra-se no apêndice deste artigo.

Tabela 9 - Emissões associadas ao processo de produção de biodiesel e hidrogênio

\begin{tabular}{|c|c|c|c|}
\hline Parâmetro(*) & UNIDADE & Valor & Fonte \\
\hline FEProdMetanol & t $\mathrm{CO}_{2 \mathrm{e} .} . \mathrm{t}$ Metanol-1 & 1,95 & (CARVALHO, 2012) \\
\hline FE ProdCatalisador & $\begin{array}{c}\mathrm{t} \mathrm{CO}_{2 \mathrm{e} . \mathrm{t}} \\
\text { Catalisador-1 }\end{array}$ & 1,2 & (CARVALHO, 2012) \\
\hline FE electrid-grid-óleo & $\mathrm{t} \mathrm{CO}_{2 \mathrm{e}} \cdot \mathrm{kWh}-1$ & 0,0001 & - \\
\hline 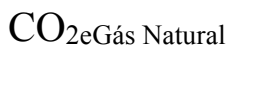 & $\begin{array}{l}\text { t CO } \mathrm{CO}_{2 \mathrm{e}} \mathrm{t} \text { Gás } \\
\text { Natural-1 }\end{array}$ & 5,600 & (QUISPE etal., 2013) \\
\hline$Q_{\text {metanol }}$ & $\begin{array}{l}\mathrm{t} \text { metanol.t } \\
\text { biodiesel-1 }\end{array}$ & 0,12 & - \\
\hline $\mathrm{Q}_{\text {catalisador }}$ & $\begin{array}{l}\mathrm{t} \text { catalisador.t } \\
\text { biodiesel-1 }\end{array}$ & 0,001 & - \\
\hline$Q_{\text {electrid-grid-bio }}$ & kWh.t biodiesel-1 & 771,45 & - \\
\hline $\mathrm{CO}_{2 \text { eprod-ins-bio }}$ & $\begin{array}{c}\mathrm{t} \mathrm{CO}_{2 \mathrm{e} . \mathrm{t}} \text { biodiesel- } \\
1\end{array}$ & $\mathbf{0 , 3 5}$ & - \\
\hline
\end{tabular}

Fonte: Própria.

(*) - A representação de cada um dos parâmetros encontra-se no apêndice deste artigo.

A Figura 2 mostra as emissões de dióxido de carbono equivalente calculadas em cada etapa do processo produtivo de biodiesel e hidrogênio.

Figura 2 - Emissões de dióxido de carbono equivalente sem considerar o ciclo do carbono obtido em cada etapa do processo de produção de biodiesel e hidrogênio 


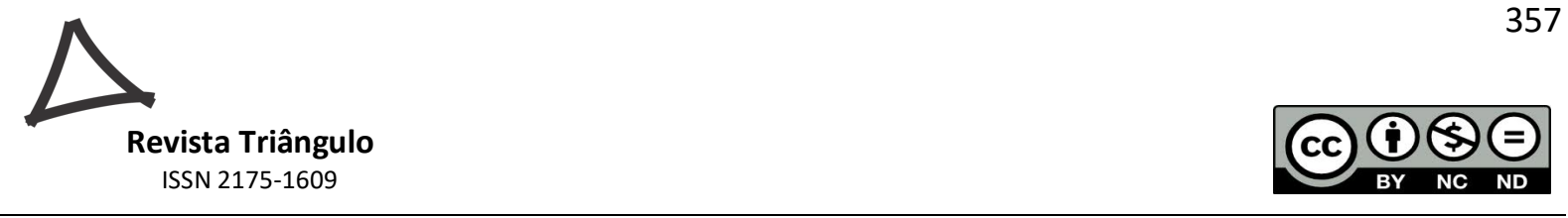

EMISSÕES DE DIÓXIDO DE CARBONO EQUIVALENTE (\%)

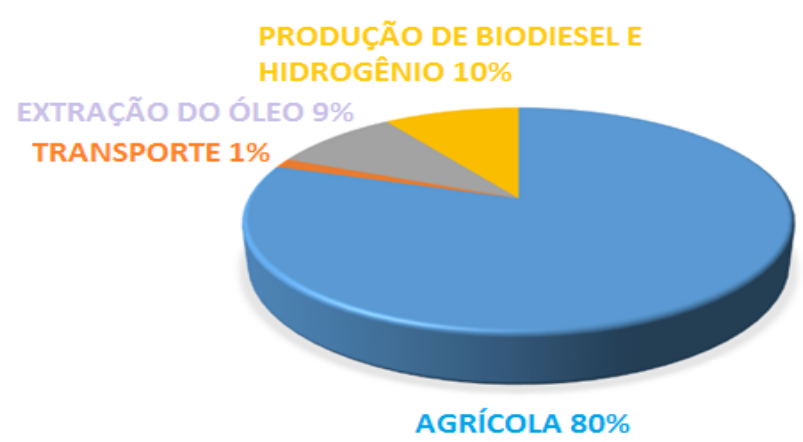

Finalmente, para computar todas as emissões, é necessário utilizar um único sistema de unidades. Como foi dito anteriormente, as emissões de dióxido de carbono equivalente são referidas a $\mathrm{kg} \mathrm{CO} 2 \mathrm{e} / \mathrm{kg}$ de óleo de canola consumido. Portanto, utilizando os fatores adimensionais de conversão definidos anteriormente, o valor total de emissões de dióxido de carbono equivalente da planta de produção de biodiesel e hidrogênio é 3,64 kg CO2e/ $\mathrm{kg}$ de óleo de canola consumido. É importante lembrar que este valor leva em conta todas as emissões, desde a etapa agrícola até a etapa industrial.

\section{CONSIDERAÇÕES FINAIS}

O estudo do impacto ambiental, em cada etapa do processo de produção integrada de biodiesel e hidrogênio, foi realizado aplicando a Metodologia de Projetos de Mecanismo de Desenvolvimento Limpo com Biodiesel da Convenção das Nações Unidas sobre Mudança do Clima, no qual foram utilizadas as equações de 1 a 5 .

A etapa associada ao transporte é a que apresenta menor emissão de $\mathrm{CO} 2 \mathrm{e}$, com valor de $1 \%$ do valor total das emissões produzidas $(3,64 \mathrm{~kg} \mathrm{CO} 2 \mathrm{e} / \mathrm{kg}$ de óleo de canola), já a etapa agrícola produz $80 \%$ do valor total das emissões, isto é devido ao consumo de fertilizantes, inseticidas e herbicidas, cujos fatores de emissão são altos quando comparados ao resto dos insumos utilizados em cada etapa do processo produtivo.

Os resultados obtidos são importantes para o desenvolvimento tecnológico da produção integrada de biodiesel e hidrogênio, de modo a procurar processos que sejam ambientalmente e energeticamente sustentáveis, isto ajudará no desenvolvimento de análises mais profundo, considerando aspectos de eficiência ecológica, indicador de poluição e ciclo do carbono da produção integrada.

\section{REFERÊNCIAS}

BARONTINI, F. et al. Pilot-scale biofuel production from sunflower crops in central Italy. Renewable Energy,vol. 83, issue C, P. 954-962, 2015. 


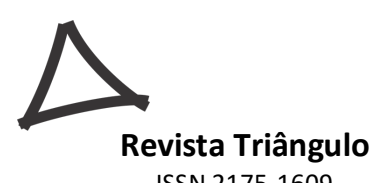

ISSN 2175-1609

BOLOY, R.A.M. Análise termoeconômica e ecológica da incorporação do processo de produção de hidrogênio em uma planta de produção de biodiesel. 2014. $133 \mathrm{f}$. Tese(Doutorado)-Universidade Estadual Paulista, Faculdade de Engenharia de Guaratinguetá, 2014.

BOLOY, R.A.M. et al. "Ecological impacts from syngas burning in internal combustion engine: Technical and economic aspects." Renewable and Sustainable Energy Reviews 15.9 (2011): 5194-5201.BRAGA, L. B. et al. Hydrogen production by biogas steam reforming: A technical, economic and ecological analysis. Renewable and Sustainable Energy Reviews, v. 28, n. 0, p. 166-173, 2013.

BURATTI, C.; MORETTI, E.; FANTOZZI, F. Assessing the GHG emissions of rapeseed and soybean biodiesel in compilance to the eurenawable energy directive methodology for biofuels. In 18th European Biomass Conference and Exhibition, Lyon, France, P. 2230$2235,2010$.

CARVAlHO, P.T. Balanço de Emissões de Gases de Efeito Estufa de Biodiesel Produzido a Partir de Soja e Dendê no Brasil. 153f. Dissertação de Mestrado em Ciências em Planejamento Energético. UFRJ/COPPE, Rio de Janeiro, 2012.

CHAWLA, S.; GHOSH, K. Thermodynamic analysis of hydrogen production from biogas for phosphoric acid fuel cell. International Journal of Hydrogen Energy, v. 17, n. 6, p. 405-412, jun. 1992.

CHEN, H. et al. Macroalgae for biofuels production: Progress and perspectives. Renewable and Sustainable Energy Reviews, v. 47, p. 427-437, 2015.

CORONADO, C. R.; VILLELA, A. DE C.; SILVEIRA, J. L. Ecological efficiency in CHP: Biodiesel case. Applied Thermal Engineering, v. 30, n. 5, p. 458-463, 2010.

GAZZONI, D.L.; BORGES, J.L.; DE AVILA, M.T; FELICI, P.H. Balanço energético da cultura da canola para a produção de biodiesel. Espaço Energia. Vol II, 2009, p. 24-28.

KLEIN, C., NOVOA, R.S.A., OGLE, S., et al. N2O Emissions From Managed Soils, and CO2 Emissions From Lime and Urea Application. In: Eggleston, S., Buendia, L., Miwa, K., Ngara, T., Tanabe, K. (eds.), 2006 IPCC Guidelines for National Greenhouse Gas Inventories - Volume 4, Capítulo 11, Prepared by the National Greenhouse Gas Inventories Programme, IGES, Japão, 2006.

LASCO, R.D., OGLE, S., RAISON, J., et al. Cropland .In: Eggleston, S., Buendia, L., Miwa, K., Ngara, T., Tanabe, K. (eds.). IPCC Guidelines for National Greenhouse Gas Inventories - Volume 4, Capítulo 5, Prepared by the National Greenhouse Gas Inventories Programme, IGES, Japão, 2006.

ONCEL, S. S. et al. Biohydrogen production from model microalgae Chlamydomonasreinhardtii: A simulation of environmental conditions for outdoor experiments. International Journal of Hydrogen Energy, v. 40, n. 24, p. 7502-7510, 2015. 
QUISPE, C. A. G.; CORONADO, C. J. R.; CARVALHO JR, J. A. Glycerol: Production, consumption, prices, characterization and new trends in combustion. Renewable and Sustainable Energy Reviews, v. 27, n. 0, p. 475-493, 2013.

SONG, M. et al. Marine brown algae: A conundrum answer for sustainable biofuels production. Renewable and Sustainable Energy Reviews, v. 50, p. 782-792, out. 2015.

UNFCCC (United Nations Framework Convention on Climate Change). Approved consolidated baseline and monitoring methodology ACM0017: Production of biodiesel for use as fuel. Version 2.0.0, 2010.

YIN, Y.; WANG, J. Biohydrogen production using waste activated sludge disintegrated by gamma irradiation. Applied Energy, v. 155, p. 434-439, out. 2010. 